\title{
Internet-based cognitive-behavioural therapy for severe health anxiety: randomised controlled trial
}

Erik Hedman, Gerhard Andersson, Erik Andersson, Brjánn Ljótsson, Christian Rück Gordon J. G. Asmundson and Nils Lindefors

\section{Background}

Hypochondriasis, characterised by severe health anxiety, is a common condition associated with functional disability. Cognitive-behavioural therapy (CBT) is an effective but not widely disseminated treatment for hypochondriasis. Internetbased CBT, including guidance in the form of minimal therapist contact via email, could be a more accessible treatment, but no study has investigated internet-based CBT for hypochondriasis.

\section{Aims}

To investigate the efficacy of internet-based CBT for hypochondriasis.

\section{Method}

A randomised controlled superiority trial with masked assessment comparing internet-based CBT $(n=40)$ over 12 weeks with an attention control condition $(n=41)$ for people with hypochondriasis. The primary outcome measure was the Health Anxiety Inventory. This trial is registrated with ClinicalTrials.gov (NCT00828152).

\section{Results}

Participants receiving internet-based CBT made large and superior improvements compared with the control group on measures of health anxiety (between-group Cohen's $d$ range $1.52-1.62)$.

\section{Conclusions}

Internet-based CBT is an efficacious treatment for hypochondriasis that has the potential to increase accessibility and availability of CBT for hypochodriasis.

\section{Declaration of interest}

None.
Hypochondriasis is an often chronic condition that is highly prevalent in medical settings and associated with an increased risk of unemployment and long-term disability. ${ }^{1-4}$ Although currently classified as a somatoform disorder according to DSM-IV, hypochondriasis is characterised by severe health anxiety and is conceptually best understood as an anxiety disorder. ${ }^{6}$ In several randomised controlled trials (RCTs), cognitive-behavioural therapy (CBT) has been shown to be effective in treating hypochondriasis $^{7-10}$ and, relative to other forms of treatment, has superior outcomes. ${ }^{11}$ However, because of scarcity of properly trained therapists and high costs, CBT is rarely available in healthcare settings. ${ }^{12}$ It is therefore essential to develop and evaluate alternative methods of delivering CBT for hypochondriasis that are as effective as conventional CBT but require less therapist time. Internet-based CBT, where treatment is delivered via the internet with online therapist guidance, seems to meet these criteria. ${ }^{13}$ To our knowledge, no study has been published on the effects of internet-based CBT for hypochondriasis and therefore the aim of the present study was to investigate this. We hypothesised that the participants receiving internet-based CBT, relative to those in an attention control condition, would show a significant reduction in health anxiety, general anxiety and depressive symptoms. In addition, we expected that participants receiving internet-based CBT would improve on measures of global functioning and quality of life.

\section{Method}

\section{Design}

This was a superiority trial within the context of a parallel group study with unrestricted randomisation in a 1:1 ratio conducted in Sweden. Outcome assessors were masked to allocation status. This trial was registered at ClinicalTrials.gov (NCT00828152).

\section{Participants and recruitment}

Participants were recruited by self-referral and by referral from psychiatrists and primary care physicians. There were no advertisements in newspapers or in other media. However, an article about the study was published in a major nationwide newspaper. In addition, information about the study was sent to all psychiatrists and primary care physicians in Stockholm County. The study was conducted at the Internet Psychiatry Clinic at the Karolinska University Hospital, Stockholm, Sweden. Information about the study was published on the official web page of the clinic. The study protocol was approved by the regional ethics committee. Informed consent was obtained from all participants.

To be eligible for inclusion, potential participants had to meet the following criteria:

(a) agree to not undergo any other psychological treatment for the duration of the study;

(b) have no history of CBT in the past 4 years;

(c) have no serious somatic disease as assessed by a physician based on a review of medical records and the anamnesis from the diagnostic assessment interview;

(d) constant dosage 2 months prior to treatment if on prescribed medication for anxiety or depression and agree to keep dosage constant throughout the study;

(e) fulfil the DSM-IV criteria of hypochondriasis as assessed using the Health Anxiety Interview; ${ }^{14}$

(f) other comorbid disorders according to the Mini International Neuropsychiatric Interview $(\mathrm{MINI})^{15}$ were allowed but hypochondriasis had to be the primary concern;

(g) not currently fulfilling the diagnostic criteria for substance misuse according to the MINI;

(h) have no history of psychosis or bipolar disorder; 
(i) not score $>20$ on the Montgomery-Åsberg Depression Rating Scale-Self-Report (MADRS-S) if criteria for major depression were met; ${ }^{16}$

(j) have a score of less than 4 of 6 on the suicide ideation item of MADRS-S; and

(k) not meet criteria for any personality disorders within cluster A or B according to the DSM-IV.

In the first stage of the recruitment process, potential participants were instructed to complete an online screen comprising the Illness Attitude Scale (IAS), ${ }^{17}$ Whiteley Index, ${ }^{18}$ Alcohol Use Disorders Identification Test (AUDIT), ${ }^{19}$ Drug Use Disorders Identification Test (DUDIT) ${ }^{20}$ and MADRS-S. Subsequently, a clinical psychologist conducted a diagnostic assessment interview via telephone to establish whether the inclusion criteria were met. To ensure reliability in the diagnostic procedure, a psychiatrist was consulted in each case. The psychiatrist reviewed the case report form, the use of pharmacotherapy and the presence of somatic disease. The psychologists conducting the assessments and the psychiatrist had more than 5 years of experience of working with structured interviews on a weekly basis and had undergone extensive training in the use of the MINI. Figure 1 shows the participant flow throughout the trial and Table 1 the demographic description of the participants.

\section{Outcome measures}

\section{Primary outcome measure}

The primary outcome measure was the Health Anxiety Inventory (HAI). ${ }^{21}$ The Short HAI (SHAI), which consists of the 18 items

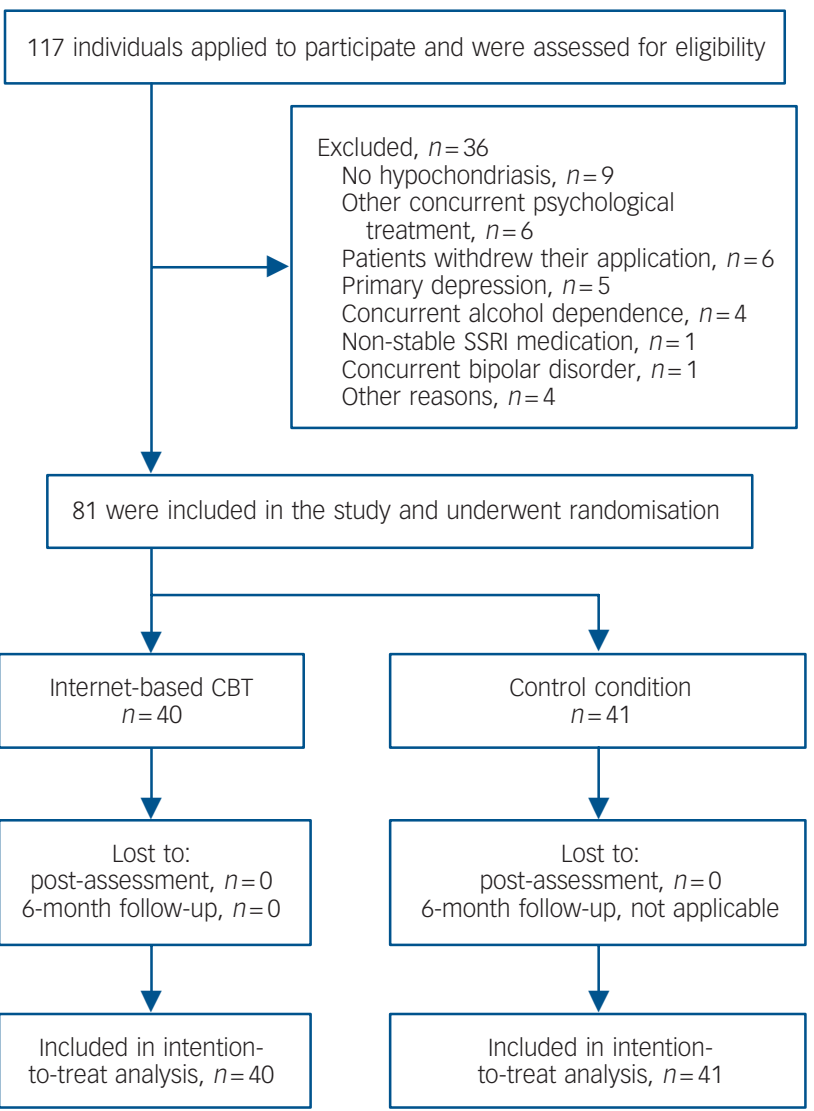

Fig. 1 Participant flow and reasons for dropping out throughout the trial.

SSRI, selective serotonin reuptake inhibitor; CBT, cognitive-behavioural therapy. most highly correlated with the full scale, was administered on a weekly basis during treatment. ${ }^{21}$

\section{Secondary outcome measures}

The IAS $^{17}$ and the Whiteley Index ${ }^{18}$ were administered as additional measures of health anxiety. We used the Beck Anxiety Inventory $(\mathrm{BAI})^{22}$ and the Anxiety Sensitivity Index (ASI) ${ }^{23}$ to assess general anxiety and anxiety sensitivity, respectively. The MADRS-S ${ }^{16}$ was used to measure depressive symptoms, and the Quality of Life Inventory (QOLI) ${ }^{24}$ to assess quality of life.

\section{Clinician-administered measures}

Psychiatric diagnoses were assessed using the Health Anxiety Interview $^{14}$ and the MINI. ${ }^{15}$ The Health Anxiety Interview is based on the Structured Clinical Interview for DSM-IV disorders $(\mathrm{SCID})^{25}$ with the added advantage of providing health anxiety information in greater detail. Global severity of psychiatric problems and functioning, respectively, were measured by the Clinical Global Impression scale $(\mathrm{CGI})^{26}$ and the Global Assessment of Functioning scale (GAF). ${ }^{5}$

\section{Procedure}

Assessments, including diagnostic interviews performed by a clinical psychologist, were conducted before treatment, immediately after treatment and 6-month follow-up. All continuous outcome measures except the GAF were administered via the internet, which has been shown to be a valid administration format for questionnaires. ${ }^{27}$ The clinical psychologists performing the assessments were masked to treatment status and when summoned to clinician assessment all participants were instructed not to mention which intervention they had received. Following interviews, the assessing

\section{Table 1 Characteristics of both groups pre-treatment}

\begin{tabular}{|c|c|c|}
\hline Variable & $\begin{array}{l}\text { Internet-based } \\
\text { cognitive-behavioural } \\
\text { therapy group } \\
(n=40)\end{array}$ & $\begin{array}{l}\text { Control } \\
\text { group } \\
(n=41)\end{array}$ \\
\hline \multicolumn{3}{|l|}{ Gender } \\
\hline Women, $n$ & 28 & 32 \\
\hline Men, $n$ & 12 & 9 \\
\hline \multicolumn{3}{|l|}{ Age } \\
\hline Age, mean (s.d.) & $39.3(9.8)$ & $38.8(9.5)$ \\
\hline Minimum-maximum, years & $25-62$ & $25-69$ \\
\hline \multicolumn{3}{|l|}{ Hypochondriasis } \\
\hline Duration: years, mean (s.d.) & $20(13.8)$ & $21.95(12.4)$ \\
\hline Age at onset, mean (s.d.) & $23.7(12.3)$ & $21.5(11.5)$ \\
\hline \multicolumn{3}{|l|}{ Occupational status } \\
\hline Working full-time, $n$ & 31 & 32 \\
\hline Unemployed, $n$ & 6 & 5 \\
\hline Sick leave, part or full-time, $n$ & 2 & 4 \\
\hline Disability pension, $n$ & 1 & 0 \\
\hline \multicolumn{3}{|l|}{ Referral, $n$} \\
\hline Self-referral & 28 & 28 \\
\hline From general practitioners & 7 & 7 \\
\hline From psychiatrists & 5 & 6 \\
\hline \multicolumn{3}{|l|}{ Stabilised medication, $n$} \\
\hline $\begin{array}{l}\text { Selective serotonin reuptake } \\
\text { inhibitor }\end{array}$ & 8 & 14 \\
\hline Serotonin and noradrenaline & & \\
\hline reuptake inhibitors & 3 & 1 \\
\hline Benzodiazepines & 1 & 1 \\
\hline Antihistamines & 2 & 1 \\
\hline
\end{tabular}


psychologists guessed allocation status for each participant. This was done to enable an analysis of the integrity of the masking.

The randomisation procedure was managed by an external person not involved in the study. A true random number service (www.random.org) was used to ensure complete randomness. The random sequence was generated after inclusion of participants to ensure that assignment of intervention was concealed from the assessing psychologists and researchers involved in the study. Participants were allocated to the internet-based CBT group or the attention control condition (control group) in a 1:1 ratio using simple randomisation. After the post-treatment assessment participants in the control group were crossed over to CBT treatment.

\section{Interventions}

\section{Internet-based CBT}

The treatment was based on a CBT model for health anxiety, emphasising the role of avoidance and safety behaviours, internal focus and interpretations of bodily sensations as signs of serious illness as maintaining factors of hypochondriasis. ${ }^{14,28}$ In addition, the treatment included mindfulness training as a means of acquiring skills to help participants expose themselves to bodily sensations without seeking reassurance. ${ }^{29}$ The basic rationale for including mindfulness was that attempts to divert attention from bodily sensations - a common strategy for people with hypochondriasis - may actually be an avoidance response that strengthens the influence of the bodily sensations and increases attention to them. ${ }^{29}$ The mindfulness training was entirely focused on teaching participants to observe their bodily sensations without trying to control them.

A central feature of the treatment was a self-help text of 118 pages, divided into 12 modules. Participants gradually gained access to the modules through an internet-based treatment platform. Each module was devoted to a specific theme and included homework exercises. The modules reflected the content of conventional CBT for hypochondriasis. ${ }^{14,28}$ The treatment protocol was developed by our research group and it has been validated in a trial investigating the effects of group CBT for hypochondriasis. ${ }^{30}$ A detailed description of each module can be found in Fig. 2.

The duration of the internet-based CBT was 12 weeks and throughout this period the participant had access to a therapist via a secure online contact system. The role of the therapist was mainly to provide feedback regarding all homework and to grant access to the succeeding treatment modules; however, the participant could contact the therapist at any time and expect a reply within $24 \mathrm{~h}$. In addition, therapists encouraged inactive participants to continue the treatment work. The participant and therapist had no face-to-face or telephone contact during the treatment. On average, therapists spent 9 min (s.d.=5.6) weekly per participant. During the treatment phase, participants also had access to an online discussion forum that enabled anonymous contact with other participants receiving internetbased CBT.

The therapists conducting the internet-based CBT were four licensed psychologists with 1-4 years of clinical experience in delivering internet-based CBT.

\section{Control condition}

The control condition consisted of an online discussion forum where participants could send messages anonymously to each over a period of 12 weeks. Online discussion forums have been shown to be effective in reducing depressive symptoms and anxiety in people with depression and breast cancer. ${ }^{31,32}$ Thus, the rationale for employing this control condition design was to ensure a basic control for attention and the possible anxiety-alleviating effect of sharing one's distress with others. Participants were encouraged to discuss their health anxiety and helpful ways of coping with it, and to provide support to others randomised to the control group. The discussion forum was monitored by a clinical psychologist on a daily basis to ensure that discussions were conducted in a respectful manner.

\section{Statistical analysis}

Statistical analyses were conducted using PASW version 18.0 for Windows (SPSS Inc, Chicago, http://support.spss.com/). The

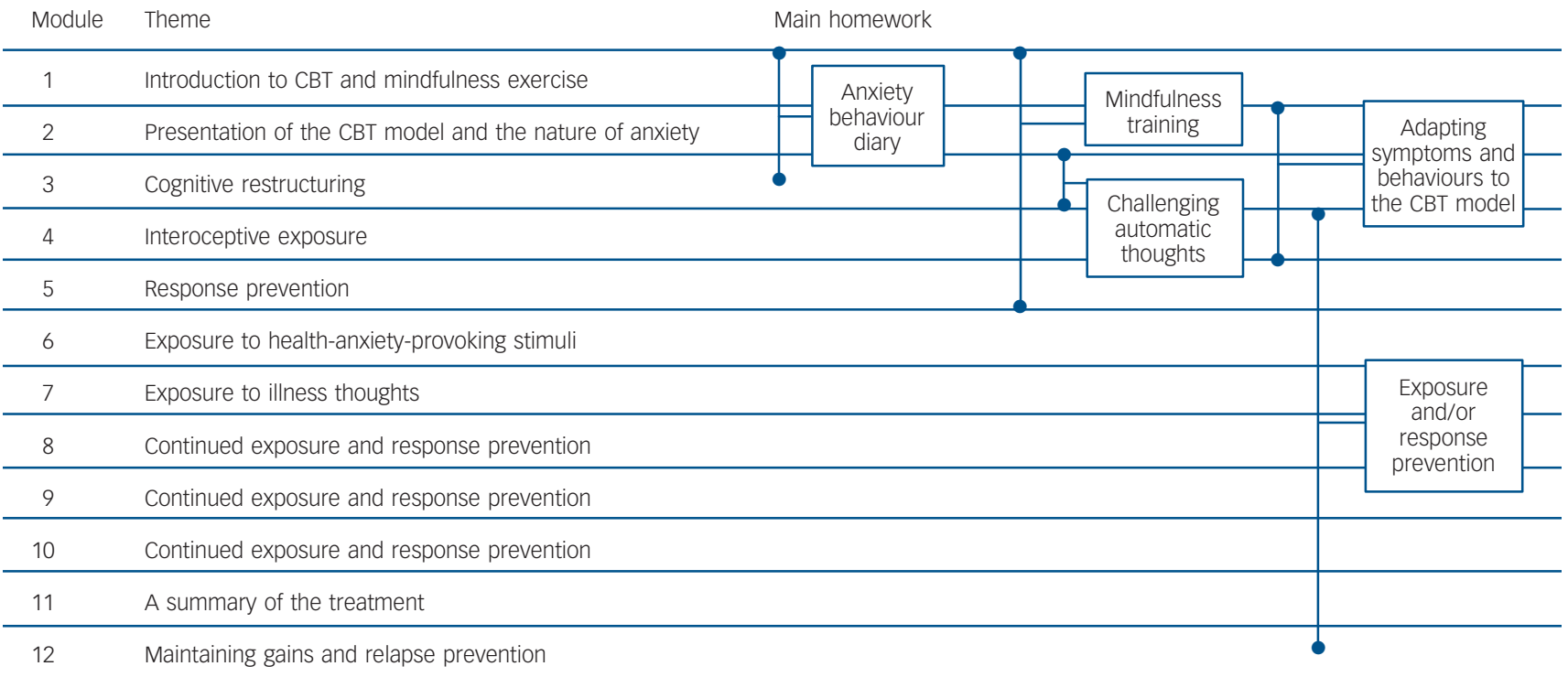


main outcome continuous variables, except SHAI, were analysed using analysis of covariance (ANCOVA) where pre-treatment scores were held as covariates. Weekly assessments of SHAI were analysed using a linear mixed-effects model because of its superior qualities regarding missing data and in reducing the risk of committing type I errors. ${ }^{33}$ We employed the restricted maximum likelihood method assuming a compound symmetry model as covariance structure since it provided the best model in an information criterion comparison. Post hoc tests for group differences each week on SHAI were conducted using $t$-tests. Since participants in the control group received internet-based CBT immediately after the post-treatment assessment, no analysis was performed on their 6-month follow-up data. Between-group analysis of nominal and ordinal scale variables were conducted using $\chi^{2}$ and Fisher's exact tests and Mann-Whitney $U$-tests, respectively. We used McNemar's tests and Wilcoxon tests to analyse within-group variables on nominal and ordinal scales, respectively. Cohen's $d$ based on pooled standard deviations was used to calculate effect sizes. The sample size was considered satisfactory since power calculations showed that there was a chance slightly lower than $80 \%$ to detect a difference, given an effect size of 0.6 and an alpha level of 0.05 .

\section{Results}

\section{Attrition and adherence}

There was no data loss at pre-treatment, post-treatment, or 6month follow-up. The average number of completed modules in internet-based CBT was 9.1 (s.d.=3.3). Six participants completed fewer than six modules and were considered non-completers. See Table 2 for the distribution of module completion. The most common reason reported for not completing treatment was lack of time. In the control group, the average number of postings was 16.1 (s.d. = 19.3). Thirty-nine of 41 participants in the control group posted at least one message.

\section{Effect sizes}

Between-group effect sizes, showing superior improvements for internet-based CBT compared with the control group, were large on measures of health anxiety at post-treatment assessment. Effect sizes, means and standard deviations for all continuous outcome measures are presented in Table 3.

\section{Primary outcome measure}

The ANCOVA for HAI difference at post-treatment assessment, holding pre-treatment values as covariates, revealed a significant effect between the internet-based CBT group and the control group $(F=95.90$, d.f. $=1,78, P<0.001)$. As illustrated in Fig. 3, the internet-based CBT group displayed continuous within-group improvements throughout the trial on the SHAI. Analysis using the mixed-effects model showed a significant interaction effect of group and time on the SHAI $(F=17.14$, d.f. $=12,823$, $P<0.001)$. Post hoc tests showed that participants in the internet-based CBT group had significantly lower scores than those in the control group from the third week onwards $(t=2.39-7.03$, d.f. $=1,60-79, P<0.001)$.

\section{Secondary outcome measures}

At post-treatment assessment, ANCOVAs showed a significant between-group effect (i.e. lower scores) for participants in the internet-based CBT group compared with those in the control group on the two secondary health anxiety measures, the IAS
$(F=92.70$, d.f. $=1,78, P<0.001)$ and Whiteley Index $(F=59.15$, d.f. $=1,78, P<0.001)$. The ANCOVAs for post-treatment change, covarying baseline scores, showed that participants in the internet-based CBT group were more improved than those in the control group on measures of depressive symptoms (MADRS-S), general anxiety (BAI), anxiety sensitivity (ASI) and quality of life (QOLI; $F=8.64-46.41$, d.f. $=1,78, P<0.004-0.001$ )

\section{Psychiatric diagnosis at each assessment point}

Following treatment, 27 of $40(67.5 \%)$ participants who had received internet-based CBT no longer met diagnostic criteria for hypochondriasis. In the control group, 2 of 41 (4.9\%) no longer met criteria for hypochondriasis. At post-treatment assessment there was a significant difference in prevalence of hypochondriasis between groups $\left(\chi^{2}=34.55\right.$, d.f. $\left.=1, P<0.001\right)$. At 6-month follow-up improvements were maintained, with 32 of 40 participants in the internet-based CBT group (80\%) no longer meeting diagnostic criteria for hypochondriasis.

\section{Clinician-administered measures of global functioning}

Compared with the control group, participants in the internetbased CBT group had significantly lower scores on the Improvement CGI-I and severity (CGI-S) subscales of the CGI at post-treatment assessment $(P<0.001)$. The distribution of CGI-I scores are illustrated in Fig. 4 . Of the 40 participants in the internet-based CBT group, 4 were classified as not at all ill $(10 \%)$ and $16(40 \%)$ as borderline mentally ill post-treatment according to the CGI-S. In the control group no participant was considered not at all ill or borderline ill at post-treatment assessment. The internet-based CBT group was further improved at follow-up as 10 participants (25\%) were classified as not at all ill and $20(50 \%)$ as borderline mentally ill $(P<0.013)$. The ANCOVA, using pre-assessment as covariate, also revealed that participants in the internet-based CBT group were significantly more improved than those in the control group on the GAF $(F=48.86$, d.f. $=1,78, P<0.001)$.

\section{Assessment of masking}

In two instances masking was broken as a consequence of the participants accidentally mentioning their treatment allocation status to the assessor. Fisher's exact test, stratified by CGI-C scores to control for differential treatment effects, showed no significant association between assessors' guess and actual treatment allocation $(P<0.58-0.052)$.

\section{Discussion}

\section{Main findings}

To our knowledge, this is the first trial aiming to investigate the efficacy of CBT delivered via the internet to people with hypochondriasis. Guided internet-based CBT resulted in superior improvements compared with the control group on all outcome variables. Following treatment, two-thirds of those in the internet-based CBT group no longer met the diagnostic criteria for hypochondriasis. Clinician-administered measures of improvement showed large effects for internet-based CBT, with treatment effects maintained at 6-month follow-up. Considering the major strengths of this study (i.e. randomisation, in-depth masked assessment, validated outcome measures), this suggests that internet-based CBT is a promising treatment alternative for hypochondriasis. The effects found in this study are comparable with previous RCTs investigating conventional CBT for health 

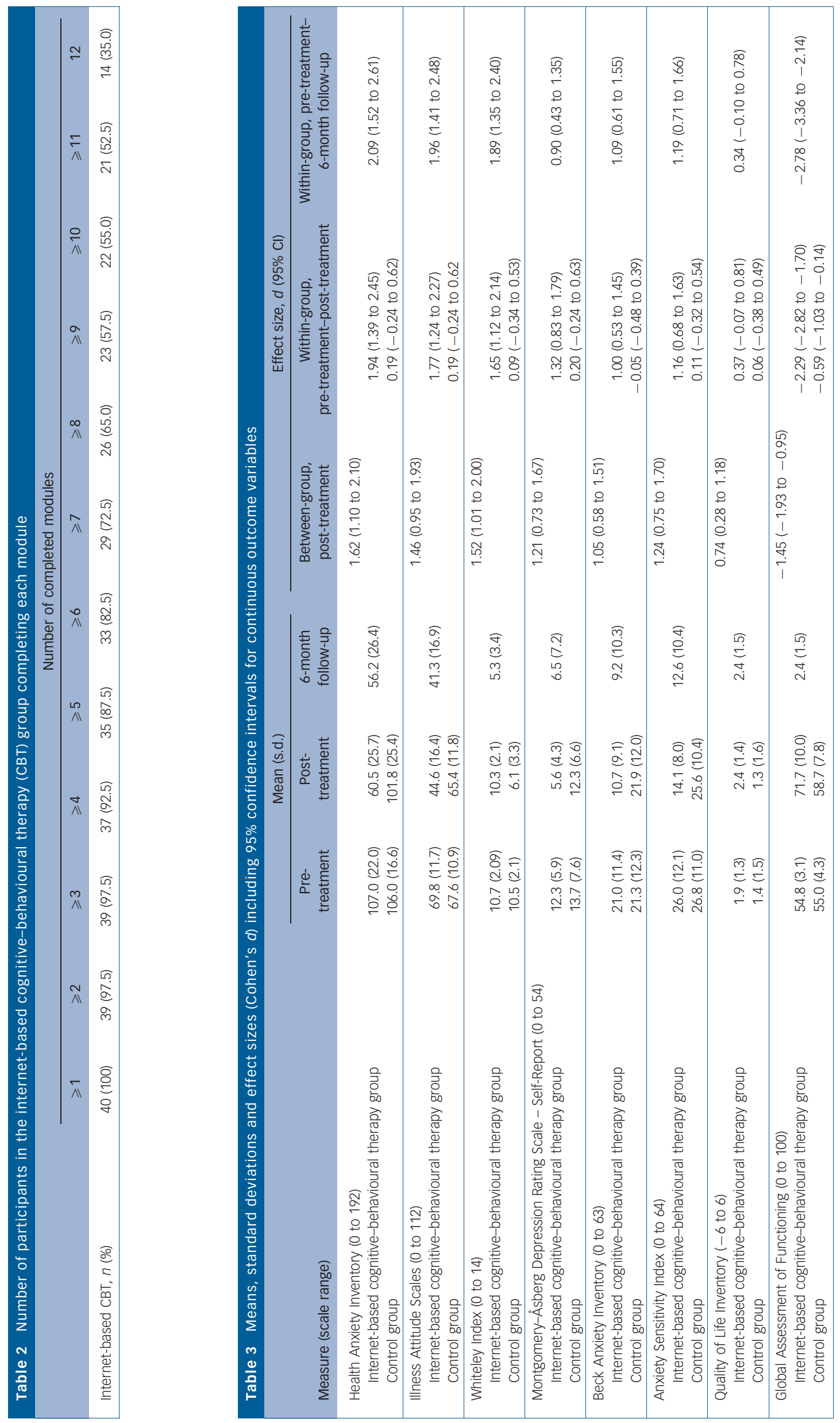


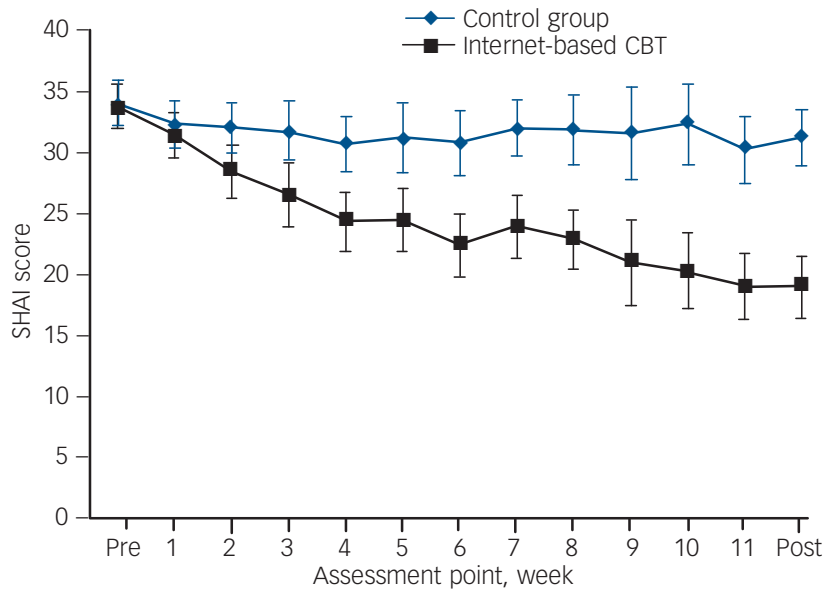

Fig. 3 Weekly assessment of Short Health Anxiety Inventory, SHAI (including 95\% confidence intervals).

CBT, cognitive-behavioural therapy.

anxiety ${ }^{5-9}$ with moderate to large improvements. Effect sizes are also comparable with those reported from trials investigating internet-based CBT for other anxiety disorders. ${ }^{34}$

\section{Treatment mechanism}

As participants in this trial suffered from hypochondriasis and made marked improvements despite no live-therapist contact a remark on treatment mechanisms is warranted. The context in which this treatment was delivered was highly controlled. Participants had to go through a thorough assessment procedure before, during and after the therapy, the caregiver was a psychiatric clinic, and the therapist was the same throughout the treatment. The most important treatment mechanisms may be the same as in conventional CBT (i.e. demanding behaviour change through processes such as exposure and response prevention). This means that, although internet-based, the main and most important part of therapy does not take place online but, rather, involves active engagement in exposing oneself to feared stimuli while refraining from using maladaptive coping strategies.

As participants' work sheets regarding exposure and response prevention were stored online, adherence to the treatment was easy to monitor during therapy. The use of mindfulness exercises with the purpose of enhancing exposure might also have contributed to the large effects observed in the present study. Previous studies have generally not included this treatment component and this could be a venue for future research.

Taken together with findings from studies of internet-based CBT for other anxiety disorders, ${ }^{35}$ the results of this study suggest that using the internet as a treatment delivery format for hypochondriasis has advantages compared with conventional CBT. Advantages include an increased sense of responsibility for the treatment and a reduced risk of therapist drift, ${ }^{36}$ which is nearly impossible in this firmly structured format of treatment delivery.

\section{Limitations}

This study has several limitations. First, the low within-group effect sizes seen in the control group indicate that the aim of having this serve as a form of active control was not achieved. Thus, it cannot be ruled out that a substantial part of the

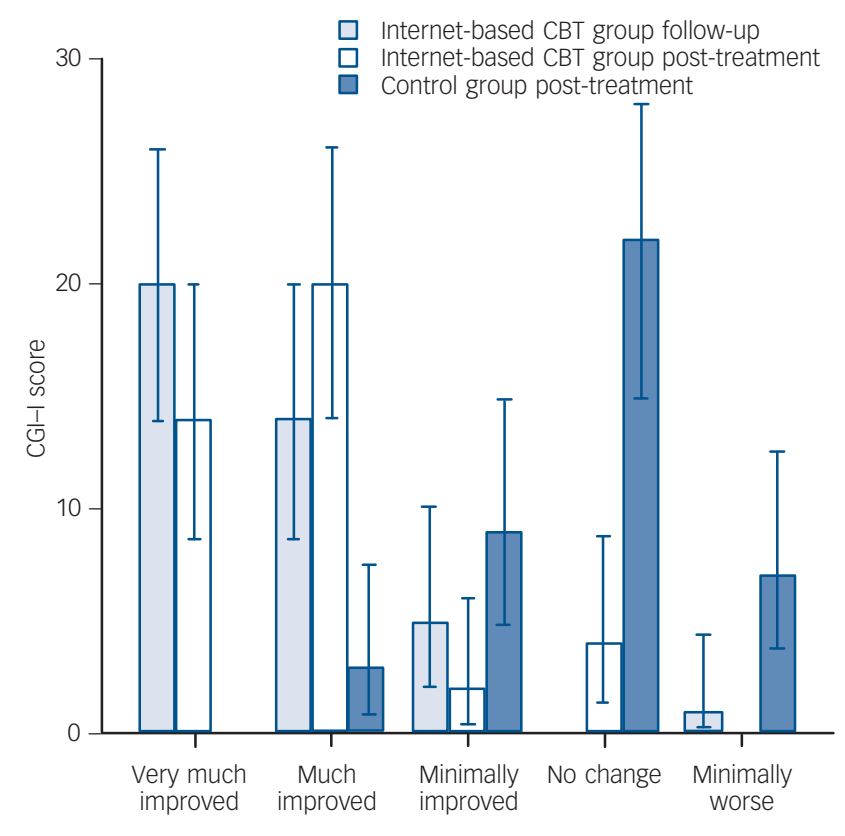

Fig. 4 Distribution of Clinical Global Impression -

Improvement (CGI-I) scores (including $95 \%$ confidence intervals) for the internet-based cognitive-behavioural therapy (CBT) and control groups at post-treatment and for the internet-based CBT group at +6 -month follow-up

treatment effect in internet-based CBT is due to non-specific factors such as increased activity and therapist attention. Second, the study design did not allow for a comparison between groups at 6-month follow-up since participants in the control group were crossed over to active treatment for ethical reasons.

\section{Implications}

In spite of these limitations, the present study suggests that it is feasible to conduct internet-based CBT for people with hypochondriasis and that this treatment approach is effective in reducing health anxiety as well as general anxiety and depressive symptoms. The minor therapist resources required, as well as the flexibility of internet-based CBT with respect to delivery, makes it a promising means for dramatically increasing the availability of CBT for hypochondriasis.

Erik Hedman, MSc, Karolinska Institutet, Department of Clinical Neuroscience, Stockholm, Sweden; Gerhard Andersson, PhD, Karolinska Institutet, Department of Clinical Neuroscience, Stockholm, and Linköping University, Department of Behavioural Sciences and Learning, Swedish Institute for Disability Research, Linköping, Sweden; Erik Andersson, MSC, Brjánn Ljótsson, MSC, Christian Rück, MD, PhD, Karolinska Institutet, Department of Clinical Neuroscience, Stockholm, Sweden; Gordon J. G. Asmundson, PhD, Department of Psychology, University of Regina, Regina, Saskatchewan, Canada; Nils Lindefors, MD, PhD, Karolinska Institutet, Department of Clinical Neuroscience, Stockholm, Sweden

Correspondence: Erik Hedman, M 46, Karolinska University Hospital Huddinge, SE-141 86 Stockholm, Sweden. Email: erik.hedman.2@ki.se

First received 8 Sep 2010, final revision 29 Oct 2010, accepted 17 Nov 2010

\section{References}

1 Noyes R Jr, Kathol RG, Fisher MM, Phillips BM, Suelzer MT, Holt CS. The validity of DSM-III-R hypochondriasis. Arch Gen Psychiatry 1993; 50: 961-70.

2 Barsky AJ, Wyshak G, Klerman GL, Latham KS. The prevalence of hypochondriasis in medical outpatients. Soc Psychiatry Psychiatr Epidemiol 1990; 25: 89-94. 
3 Faravelli C, Salvatori S, Galassi F, Aiazzi L, Drei C, Cabras P. Epidemiology of somatoform disorders: a community survey in Florence. Soc Psychiatry Psychiatr Epidemiol 1997; 32: 24-9.

4 Barsky AJ, Fama JM, Bailey ED, Ahern DK. A prospective 4- to 5-year study of DSM-III-R hypochondriasis. Arch Gen Psychiatry 1998; 55: 737-44.

5 American Psychiatric Association. Diagnostic and Statistical Manual of Mental Disorders (4th edn, revised) (DSM-IV-TR). APA, 2000

6 Olatunji BO, Deacon BJ, Abramowitz JS. Is hypochondriasis an anxiety disorder? Br J Psychiatry 2009; 194: 481-2.

7 Seivewright $H$, Green J, Salkovskis $P$, Barrett B, Nur U, Tyrer P. Cognitivebehavioural therapy for health anxiety in a genitourinary medicine clinic: randomised controlled trial. Br J Psychiatry 2008; 193: 332-7.

8 Greeven A, van Balkom AJ, Visser S, Merkelbach JW, van Rood YR, van Dyck $\mathrm{R}$, et al. Cognitive behavior therapy and paroxetine in the treatment of hypochondriasis: a randomized controlled trial. Am J Psychiatry 2007; 164: 91-9.

9 Clark DM, Salkovskis PM, Hackmann A, Wells A, Fennell M, Ludgate J, et al. Two psychological treatments for hypochondriasis. A randomised controlled trial. Br J Psychiatry 1998; 173: 218-25.

10 Warwick HMC, Clark DM, Cobb AM, Salkovskis PM. A controlled trial of cognitive-behavioural treatment of hypochondriasis. Br J Psychiatry 1996 169: 189-95.

11 Taylor S, Asmundson GJG, Coons MJ. Current directions in the treatment of hypochondriasis. J Cog Psychother 2005; 19: 285-304.

12 Shapiro DA, Cavanagh K, Lomas H. Geographic inequity in the availability of cognitive behavioural therapy in England and Wales. Behav Cogn Psychother 2003; 31: 185-92.

13 Andersson G. Using the internet to provide cognitive behaviour therapy. Behav Res Ther 2009; 47: 175-80.

14 Taylor S, Asmundson GJG. Treating Health Anxiety: A Cognitive-Behavioral Approach. Guilford Press, 2004.

15 Sheehan DV, Lecrubier $\mathrm{Y}$, Sheehan $\mathrm{KH}$, Amorim $\mathrm{P}$, Janavs J, Weiller $\mathrm{E}$, et al. The mini-international neuropsychiatric interview (M.I.N.I.): the development and validation of a structured diagnostic psychiatric interview for DSM-IV and ICD-10. J Clin Psychiatry 1998; 59 (suppl 20): 22-33.

16 Svanborg P, Åsberg M. A new self-rating scale for depression and anxiety states based on the comprehensive psychopathological rating scale. Acta Psychiatr Scand 1994; 89: 21-8.

17 Speckens AE, Spinhoven $\mathrm{P}$, Sloekers PP, Bolk JH, van Hemert AM. A validation study of the Whitely index, the illness attitude scales, and the somatosensory amplification scale in general medical and general practice patients. J Psychosom Res 1996; 40: 95-104.

18 Pilowsky I. Dimensions of hypochondriasis. Br J Psychiatry 1967; 113: 89-93.

19 Saunders JB, Aasland OG, Babor TF, de la Fuente JR, Grant M. Development of the alcohol use disorders identification test (AUDIT): WHO collaborative project on early detection of persons with harmful alcohol consumption. Addiction 1993; 88: 791-804.
20 Berman AH, Bergman H, Palmstierna T, Schlyter F. Evaluation of the drug use disorders identification test (DUDIT) in criminal justice and detoxification settings and in a Swedish population sample. Eur Addict Res 2005; 11 22-31.

21 Salkovskis PM, Rimes KA, Warwick HM, Clark DM. The health anxiety inventory: development and validation of scales for the measurement of health anxiety and hypochondriasis. Psychol Med 2002; 32: 843-53.

22 Beck AT, Epstein N, Brown G, Steer RA. An inventory for measuring clinical anxiety: psychometric properties. J Consult Clin Psychol 1988; 56: 893-7.

23 Reiss S, Peterson RA, Gursky DM, McNally RJ. Anxiety sensitivity, anxiety frequency and the prediction of fearfulness. Behav Res Ther 1986; 24: 1-8

24 Frisch MB, Cornell J, Villanueva M, Retzlaff PJ. Clinical validation of the quality of life inventory. A measure of life satisfaction for use in treatment planning and outcome assessment. Psychol Assess 1992; 4: 92-101.

25 First MB, Spitzer RL, Gibbon M, Williams JBW. Structured Clinical Interview for DSM-IV-TR Axis I Disorders: Research Version, Patient Edition (SCID-I/P). Biometrics Research, 2002

26 Guy W. Clinical Global Impressions. US Department of Health and Human Services, 1976.

27 Ritter $\mathrm{P}$, Lorig $\mathrm{K}$, Laurent $\mathrm{D}$, Matthews $\mathrm{K}$. Internet versus mailed questionnaires: a randomized comparison. J Med Internet Res 2004; 6: e29.

28 Furer $\mathrm{P}$, Walker JR, Stein MB. Treating Health Anxiety and Fear of Death: A Practitioner's Guide. Springer, 2007

29 McCracken LM. "Attention" to pain in persons with chronic pain: a behavioral approach. Behav Ther 1997; 28: 271-84.

30 Hedman E, Ljótsson B, Andersson E, Rück C, Andersson G, Lindefors N. Effectiveness and cost offset analysis of group CBT for hypochondriasis delivered in a psychiatric setting - an open trial. Cogn Behav Ther 2010; Oct 22 (Epub ahead of print).

31 Winzelberg AJ, Classen C, Alpers GW, Roberts H, Koopman C, Adams RE, et al. Evaluation of an internet support group for women with primary breast cancer. Cancer 2003; 97: 1164-73.

32 Houston TK, Cooper LA, Ford DE. Internet support groups for depression: a 1-year prospective cohort study. Am J Psychiatry 2002; 159: 2062-8.

33 Gueorguieva R, Krystal JH. Move over ANOVA. Progress in analyzing repeated-measures data and its reflection in papers published in the archives of general psychiatry. Arch Gen Psychiatry 2004; 61: 310-7.

34 Spek V, Cuijpers P, Nyklícek I, Riper H, Keyzer J, Pop V. Internet-based cognitive behaviour therapy for symptoms of depression and anxiety: a meta-analysis. Psychol Med 2007; 37: 319-28.

35 Andersson G, Bergstrom J, Carlbring P, Lindefors $\mathrm{N}$. The use of the internet in the treatment of anxiety disorders. Curr Opin Psychiatry 2005; 18: 73-7.

36 Waller G. Evidence-based treatment and therapist drift. Behav Res Ther 2009; 47: 119-27.

The European Convention on Human Rights is a living instrument evolving with societal changes. Human rights can appear nebulous concepts, especially in the media. People know they have them but do not know their intricacies. Oscar Wilde, in his play Vera, or the Nihilists (1880), prophetically encapsulates this: 'In the old times men carried out their rights for themselves as they lived, but nowadays every baby seems born with a social manifesto in its mouth much bigger than itself'. However, fairness, respect, equality, dignity and autonomy, which form a human rights-based approach, should be ubiquitous concepts easily applied in practice. 